\title{
A Scanning Electron Microscope Evaluation of the Efficacy of Different Fluoride-releasing Dental Restorative Materials to Prevent Enamel Demineralization: An In Vitro Study
}

\author{
Kiran M Dhananjaya ${ }^{1}$, Mrinmoy Chakraborty ${ }^{2}$, Suneel V Vadavadagi ${ }^{3}$, Garima Sinha ${ }^{4}$, Tanya Verma ${ }^{5}$, Saikat Deb ${ }^{6}$
}

\begin{abstract}
Aim: Aim of the present research was to investigate the effectiveness of various fluoride-releasing dental restorative agents in preventing demineralization of enamel.

Materials and methods: Eighty human mandibular permanent molar teeth constituted the study group. All samples were subjected to storage in thymol, after which they were taken out to prepare alike proximal box in each. Inductions of artificial enamel surface lesions were done by placing the teeth in demineralizing solution for 96 hours. Subsequently, all 80 molars were randomly assigned to any of the four groups (i.e., 20 in every individual group) according to the restoration as group A: giomer (composite resin containing surface pre-reacted glass-ionomer fillers), group B: compomer (polyacid-modified composite resin), group C: resin-modified glass-ionomer cement (RMGIC), group D: fluoride-releasing composite. After this, the $\mathrm{pH}$ cycling was performed, and the samples were subjected to examination beneath scanning electron microscope (SEM).

Results: Higher mean areas of remineralization were noted when RMGIC (96.34 \pm 0.06$)$ was used followed by the compomer (109.52 \pm 0.17$)$, giomer (118.39 \pm 0.82$)$, and the fluoride-releasing composite group $(129.27 \pm 0.31)$ in that order. A statistically significant difference was seen amid the investigational groups that utilized different restorative agents $(p<0.001)$. A pairwise evaluation that was performed revealed that except for the giomer group and the compomer group, a statistically significant difference $(p<0.001)$ was found among the experimental groups. Conclusion: This research infers that the RMGIC-treated samples exhibited significantly superior performance in preventing enamel demineralization in comparison to compomer, giomer as well as fluoride-releasing composites.

Clinical significance: One among the highly frequently employed anticariogenic materials is fluorides. Owing to this characteristic, they are integrated into numerous restorative substances. Nevertheless, the quantity and speed of fluoride release differ in different agents, which translates to the efficacy of the restorative agent in avoiding demineralization about the restoration.

Keywords: Demineralization, Fluoride, Remineralization, Restorative materials.

The Journal of Contemporary Dental Practice (2021): 10.5005/jp-journals-10024-3188
\end{abstract}

\section{INTRODUCTION}

The significant part played by fluoride in preventing dental decay is well known since the 1930s when it was noted that patients with fluorosis had a lesser amount of dental caries. In the ensuing decades, this matter has been extensively dealt with stabling that fluoride exhibits a topical action instead of acting systemically. It has been documented that prolonged contact of the dentition to fluoride is a rather efficient method to utilize its topical effect in reducing dental decay. ${ }^{1}$

Among the frequently encountered disorders with high prevalence is the dental carious process that occurs in human beings globally. Dental decay is a microbial disorder of teeth distinguished by localized annihilation of the tooth structure. Varied microbial flora house within saliva and can generate acids on the surface of natural teeth with consequent demineralization of the dental tissues. ${ }^{2}$ The demineralizing process begins as a tiny lesion that progresses inwards towards the dental tissues. If the decay process touches the pulp, necrosis of pulp tissues can occur. Furthermore, the infectious process can advance through the periodontal tissues across the apical part of the root leading to a periapical abscess. Dental caries have a multifactorial etiology, despite this certain key factors that affect dental caries are saliva and dentition of the host, microbial flora that generate acids on the surface of teeth as well as consuming a diet rich in fermentable carbohydrates. ${ }^{3}$
1,2,4,5 Department of Conservative Dentistry and Endodontics, Vananchal Dental College and Hospital, Garhwa, Jharkhand, India ${ }^{3}$ Department of Prosthodontics, SJM Dental College and Hospital, Chitradurga, Karnataka, India

${ }^{6}$ Department of Prosthodontics, Crown Bridge and Implantology, Awadh Dental College and Hospital, Jamshedpur, Jharkhand, India

Corresponding Author: Suneel V Vadavadagi, Department of Prosthodontics, SJM Dental College and Hospital, Chitradurga, Karnataka, India, Phone: +91 9845804642, e-mail: Sunilvvv123@gmail.com

How to cite this article: Dhananjaya KM, Chakraborty $M$, Vadavadagi SV, et al. A Scanning Electron Microscope Evaluation of the Efficacy of Different Fluoride-releasing Dental Restorative Materials to Prevent Enamel Demineralization: An In Vitro Study. J Contemp Dent Pract 2021;22(11):1292-1296.

Source of support: Nil

Conflict of interest: None

Restorations aid in repairing cavitations in teeth that have been destroyed by dental decay. Current dental materials have been formulated to offer resistance to secondary caries as well as prevent marginal microleakage due to the capability they have from the dissemination of fluorides and bonding to the prepared

() The Author(s). 2021 Open Access This article is distributed under the terms of the Creative Commons Attribution 4.0 International License (https://creativecommons. org/licenses/by-nc/4.0/), which permits unrestricted use, distribution, and non-commercial reproduction in any medium, provided you give appropriate credit to the original author(s) and the source, provide a link to the Creative Commons license, and indicate if changes were made. The Creative Commons Public Domain Dedication waiver (http://creativecommons.org/publicdomain/zero/1.0/) applies to the data made available in this article, unless otherwise stated. 
tooth. Restorative margins are typically significant and their loss of integrity can appreciably enhance the menace of secondary caries. ${ }^{4}$

In an attempt to provide shielding from recurrent dental decay, restorative agents that release fluoride have been formulated. Among them, the glass-ionomer cements (GICs) coupled with their hybrids, such as resin-modified glass-ionomer cement (RMGIC), polyacid altered composite resins, and composite resin having surface pre-reacted glass-ionomer (S-PRG) fillers are of key significance. Through the release of fluorides, they secure the dental hard tissues as well as the adjacent microbial atmosphere. ${ }^{5}$ Glass-ionomer cements and composite exhibit a promising future in the field of preventive restorative dentistry and appear to fulfill the demands of an ideal restorative material possessing adequate anticariogenic properties. Only limited information in the literature is available regarding the efficacy of these materials in preventing incipient interproximal lesions. Therefore, the current research was performed to investigate the effect of the fluoride-releasing restorative agents in preventing the demineralization of the enamel surface.

\section{Materials and Methods}

The current in vitro research was performed in the Department of Prosthodontics, SJM Dental College and Hospital, Chitradurga, India. Ethical approval was obtained from the institutional review board.

\section{Collection and Preparation of Samples}

Eighty permanent mandibular molars extracted due to periodontal problems were gathered from the oral surgery department and informed consent was taken. Following removal, every tooth was subjected to scrubbing and cleansing. Sound teeth with no fracture or crack, no occlusal/proximal restoration were included in the present study. Developmental imperfections, enamel cracks, WSL's as well as dental caries were excluded from the study. All the molars were subjected to storage in thymol and preserved till the research begun. Prior to use, the molars were subjected to washing beneath water followed by cleansing in an aqueous pumice slurry employing a handpiece/rubber cup.

\section{Preparation of Proximal Box}

All the molars that were preserved in thymol were taken out and an alike proximal box preparation that measured $2.0 \mathrm{~mm}$ mesiodistally, $2 \mathrm{~mm}$ occlusogingivally as well as $2 \mathrm{~mm}$ in-depth on the contact enamel surface utilizing a diamond bur fixed in a high-speed handpiece with steady water spray. The cavity dimensions were homogenized employing a divider, calibrated scale as well as a periodontal probe. The bur was kept at $90^{\circ}$ to the surface of the tooth to result in a cavosurface angle that was approximately $90^{\circ}$.

\section{Demineralization Process}

A replica of the demineralizing solution was prepared using a mix of analytical state chemical agents. The components of the solution were potassium dihydrogen phosphate $\left(\mathrm{KH}_{2} \mathrm{PO}_{4} \cdot 7 \mathrm{H}_{2} \mathrm{O}\right) 2.2 \mathrm{mmol} / \mathrm{L}$, lactic acid $0.05 \mathrm{mmol} / \mathrm{L}$ plus calcium chloride $\left(\mathrm{CaCl}_{2} \cdot 2 \mathrm{H}_{2} \mathrm{O}\right)$ $2.2 \mathrm{mmol} / \mathrm{L}{ }^{6} \mathrm{pH}$ was finalized to 4.5 by the addition of acetic acid buffer. The samples were kept in the demineralizing solution for 96 hours for induction of artificial lesions on the enamel surface.

Subsequent to the demineralizing procedure, the 80 molars were randomly assigned to any of the four groups (20 in every group) depending on the restoration used as

Group A: Giomer (composite resin containing S-PRG fillers): (Beautifil II, Shofu; Kyoto, Japan)

Group B: Compomer (polyacid-modified composite resin): (Dyract AP, Dentsply, Konstanz, Germany)

Group C: RMGIC: (3M ESPE; St Paul, Minnesota, USA)

Group D: Fluoride-releasing composite: (Unifil flow, GC, Japan)

\section{$\mathrm{pH}$ Cycling Process}

After the process of demineralization followed by positioning the restoration in the sample teeth, they were rinsed using distilled water for 60 seconds. After this, the samples were placed in $50 \mathrm{~mL}$ of artificial saliva solution. A mix of analytical state chemicals plus distilled water was employed to organize artificial saliva. The artificial saliva was prepared using the following-potassium dihydrogen phosphate $\left(\mathrm{KH}_{2} \mathrm{PO}_{4}\right) 0.9 \mathrm{mmol} / \mathrm{L}$, potassium chloride (KCl) $50 \mathrm{mmol} / \mathrm{L}$, calcium chloride $\left(\mathrm{CaCl}_{2}\right) 1.5 \mathrm{mmol} / \mathrm{L}$, as well as tris buffer $20 \mathrm{mmol} / \mathrm{L} .{ }^{6}$ After this, the samples were subjected to washing beneath distilled water. Two investigators were involved in the research. But one trained and calibrated investigator was performed the restorative procedure to maintain the uniformity of restoration.

\section{Evaluation of Samples under Scanning Electron Microscope}

Prior to assessment of the samples beneath scanning electron microscope (SEM), the teeth were coated using 40-60 nm of gold employing a sputter coater. This was pursued by evaluation beneath the SEM (Carl Zeiss EVO 40) at 2,000 $\times$ magnification (Figs $1 \mathrm{~A}$ to D). Scoring was then noted as per the criteria mentioned under: ${ }^{7}$
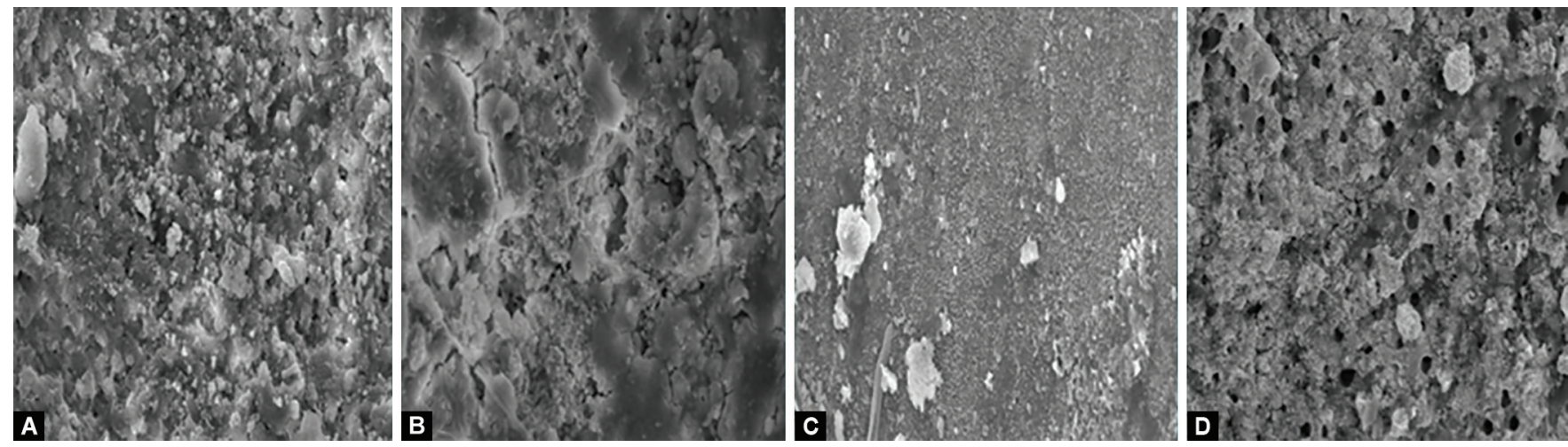

Figs 1A to D: SEM images of (A) Giomer; (B) Compomer; (C) RMGIC; (D) Fluoride-releasing composite 
Score 0-enamel surface continued perfectly integral devoid of grooves, pits/pores

Score 1-the presence of enamel surface irregularities, in the absence of demineralization of prismatic and/or interprismatic enamel

Score 2-the presence of creases and demineralized regions of prismatic/interprismatic enamel

Score 3-disseminated demineralization concerning the rod core, with the collapse of prism morphology

\section{Statistical Analysis}

SPSS version 20.0 was used to analyze the data statistically. The standard deviation and mean were calculated. The evaluation of the outcome of fluoride-releasing restorations in lessening the enamel surface demineralization was measured using the one-way analysis of variance. A $p$ value less than 0.05 was considered statistically significant.

\section{Results}

Table 1 depicts the evaluation of the mean demineralization depth amid various restorative agent groups. Giomer group depicted the higher $(142.72 \pm 0.21)$ mean demineralized regions, followed by fluoride-releasing composites $(142.03 \pm 0.17)$, the RMGIC group $(141.98 \pm 0.27)$, and the compomer group $(141.05 \pm 0.09)$, respectively. This difference among the study groups was not statistically significant.

Table 2 delineates the evaluation of the mean remineralization depth amid restorative agent groups. Higher mean remineralization regions were noted when using RMGIC $(96.34 \pm 0.06)$, followed by compomer (109.52 \pm 0.17$)$, giomer $(118.39 \pm 0.82)$ as well as the

Table 1: Assessment of the mean demineralization depth among different restorative material groups

\begin{tabular}{lcccc}
\hline Restorative materials & $n$ & Mean $\pm S D\left(\mu m^{2}\right)$ & $F$ & pvalue \\
\hline Group A: giomer & 20 & $142.72 \pm 0.21$ & & \\
Group B: compomer & 20 & $141.05 \pm 0.09$ & & \\
Group C: RMGIC & 20 & $141.98 \pm 0.27$ & 26.914 & 0.216 \\
$\begin{array}{l}\text { Group D: fluoride- } \\
\text { releasing composite }\end{array}$ & 20 & $142.03 \pm 0.17$ & & \\
\hline
\end{tabular}

Table 2: Assessment of the mean remineralization depth among different restorative material groups

\begin{tabular}{lcccc}
\hline Restorative materials & $n$ & Mean $\pm S D\left(\mu m^{2}\right)$ & $F$ & pvalue \\
\hline Group A: giomer & 20 & $118.39 \pm 0.82$ & & \\
Group B: compomer & 20 & $109.52 \pm 0.17$ & & \\
Group C: RMGIC & 20 & $96.34 \pm 0.06$ & 26.148 & 0.001 \\
$\begin{array}{l}\text { Group D: fluoride- } \\
\text { releasing composite }\end{array}$ & 20 & $129.27 \pm 0.31$ & & \\
\hline
\end{tabular}

fluoride-releasing composite $(129.27 \pm 0.31)$, respectively. This difference among the study groups using different restorations was statistically significant ( $p<0.001$ ).

Comparative assessment of areas of demineralization as well as remineralization amid the investigational groups is depicted in Table 3. RMGIC use showed the highest area of remineralization distinction with a mean of $45.64 \pm 0.21$ pursued by compomer ( $31.53 \pm 0.08)$, giomer $(24.33 \pm 0.61)$ as well as fluoride-releasing composite $(12.76 \pm 0.14)$ in that order. This difference among the study groups was statistically significant.

Table 4 presents the pairwise comparative assessment of remineralization area dissimilarity amid restorative agents using the Tukey HSD. Except for the giomer group and the compomer group, a statistically significant distinction $(p<0.001)$ was found among the experimental groups.

\section{Discussion}

The preliminary phase of the development of dental caries is characterized by the demineralization of enamel. The solution to enduring prevention and control of dental caries is remineralization. Restorative agents are being reinforced with fluorides as they have the distinctive ability to produce fluorapatite crystals and help enamel resist an acidic breakdown as well as ensuing demineralization. The quantity and structure of fluoride differ in diverse agents which decides the amount of fluoride release. A continued fluoride discharge, as well as a close approximation of the restorative margin to the tooth surface, is required to aid the integration of fluoride within the hydroxyapatite of the adjoining enamel. ${ }^{8}$

The agents consisting of fluoride which liberated quantifiable amounts of fluoride in the 7-day experimental duration were assessed in this research. But, there was a considerable difference in the quantity of fluoride discharged. The release of fluoride ions is a complicated procedure. It may be influenced by various inherent parameters like the formation of an organic matrix as well as fillers, a quantity of intrinsic/additional fluoride, and the porosity/dissolution capability of the agent's used. ${ }^{9}$ Extrinsic factors like $\mathrm{pH}$, environmental temperature, regularity of storage solution change, plaque plus pellicle development, and the sort of storage medium used. ${ }^{10}$ Also, the powder:liquid ratio employed in the preparation of the agent and the technique of mixing, duration of cure, and uncovered surface area of the agent can all influence fluoride liberation. ${ }^{11}$

Glass-ionomer cements as well as composite resins display a hopeful potential in the domain of preventive restorative dentistry as they apparently accomplish the properties that an idyllic restorative material must possess like ample anticaries characteristics. ${ }^{12}$ Research by Dionysopoulos et al. ${ }^{13}$ depicts significantly lower demineralization about the restorative material as well as absent recurrent enamel lesions in teeth restored using GICs in comparison to teeth restored with composite. Nonetheless,

Table 3: Area of demineralized and remineralization comparison among study groups

\begin{tabular}{|c|c|c|c|c|c|}
\hline Restorative materials & Demineralized area $\left(\mu m^{2}\right)$ & Remineralized area $\left(\mu m^{2}\right)$ & Mean area difference $\left(\mu m^{2}\right)$ & $F$ & $p$ value \\
\hline Group A: giomer & $142.72 \pm 0.21$ & $118.39 \pm 0.82$ & $24.33 \pm 0.61$ & \multirow{4}{*}{11.184} & \multirow{4}{*}{0.001} \\
\hline Group B: compomer & $141.05 \pm 0.09$ & $109.52 \pm 0.17$ & $31.53 \pm 0.08$ & & \\
\hline Group C: RMGIC & $141.98 \pm 0.27$ & $96.34 \pm 0.06$ & $45.64 \pm 0.21$ & & \\
\hline Group D: fluoride-releasing composite & $142.03 \pm 0.17$ & $129.27 \pm 0.31$ & $12.76 \pm 0.14$ & & \\
\hline
\end{tabular}


Table 4: Pairwise comparative evaluation of remineralized area difference among restorative materials

\begin{tabular}{llcl}
\hline \multirow{4}{*}{ Group } & Compared with & $\begin{array}{c}\text { Mean difference } \\
(I-J)\end{array}$ & Sig. \\
\hline \multirow{4}{*}{ Giomer } & Compomer & 8.87 & 0.261 \\
& RMGIC & 22.05 & 0.001 \\
& Fluoride-releasing & -10.88 & 0.04 \\
& composite & & \\
Compomer & Giomer & -8.87 & 0.261 \\
& RMGIC & 13.18 & 0.02 \\
& Fluoride-releasing & -19.75 & 0.001 \\
RMGIC & composite & & \\
& Giomer & -22.05 & 0.001 \\
Fluoride-releasing & Compomer & -13.18 & 0.02 \\
composite & Fluoride-releasing & -32.93 & 0.001 \\
& Composite & & \\
& Compomer & 10.88 & 0.04 \\
& RMGIC & 19.75 & 0.001 \\
& & 32.93 & 0.001 \\
\hline
\end{tabular}

in vitro analyses by Temin ${ }^{14}$ show considerable fluoride pickup by the contiguous enamel, rendering proof that they have anticarious potential.

The current research inferred that higher mean regions of remineralization were noted with RMGIC, followed by compomer, giomer, and finally the fluoride-disseminating composites in that order. This finding is in harmony with the research by Wiegand et al., ${ }^{15}$ Preston et al., ${ }^{16}$ Takahashi et al. ${ }^{17}$ who have stated that RMGIC has the ability to liberate fluoride ions efficiently. This has been pointed out to the infiltrating fluoride ions within the cement matrix instead of fluoride adsorption onto the enamel. The recharging capabilities of materials crucially depend on the porosity and permeability of GICs which in turn influence the recharging and re-liberation of fluoride ions from GIC.

Less liberation of fluoride from compomer could be attributed to the point that they are hydrophobic resins setting via polymerization. They do not consist of water, as is the case of resin-modified glass ionomers, although they absorb water following weeks subsequent to curing thus creating a secondary acid-base reaction, and hence the quantity of fluoride disseminated by them is lower. ${ }^{18}$

A compomer-Dyract extra comprises a mix of monomers as well as reactive glass fillers consisting of $\mathrm{SrF}_{2}$. The preliminary setting occurs by photopolymerization, which is pursued by an acid-base reaction arising from water sorption. ${ }^{19}$ Beautifil II (giomer) likewise also is composed of fluoridated glass fillers plus a glass ionomer matrix layer. Differing from compomers, fluoroalumino-silicate glass particles are made to react with polyacrylic acid before incorporation into the resin matrix. ${ }^{20}$ Fluoride levels that are reached by fluoride-releasing composites are significantly lesser than those liberated by RMGICs. This can be probably attributed to an absent acid-base reaction and less primary fluoride quantity.

Tantbirojn et al. ${ }^{21}$ state that the inhibition of enamel demineralization has been shown to occur in vitro at distances of up to $7 \mathrm{~mm}$ from RMGIC restorations. Another study by Ferracane et al. ${ }^{22}$ reported that the degree of protection was highest in close vicinity to the restorations and that the depth of lesions increased with the distance and was inversely associated with the level of fluoride released. In this study, the degree of protection offered by the fluoride-releasing materials tested was greater near the material. Yip and Smales ${ }^{23}$ explain that this potential in RMGIC may be affected not only by the formation of complex fluoride compounds and their interaction with polyalkenoate acid but also by the type and amount of resin used for the photochemical polymerization reaction.

Limitation of this research-although this research has attempted to replicate the clinical situations, an in vitro experimentation cannot simulate oral settings entirely. The mock caries research quantifies just resistance of enamel/dentin to endure acid insults. The consequence of bacterial attacks, demineralization, and remineralization cycles as well as the likelihood for recharging with outside sources of fluoride in the clinical setup were not evaluated. Thus, it is important to carry out a clinical examination to compare with in vitro trials in an effort to choose an ideal restoration.

\section{Conclusion}

This research infers that the RMGIC-treated samples exhibited significantly superior performance in preventing enamel demineralization in comparison to compomer, giomer as well as fluoride-releasing composites.

\section{References}

1. Ten Cate JM. Contemporary perspective on the use of fluoride products in caries prevention. Br Dent J 2013;214(4):161-167. DOI: 10.1038/sj.bdj.2013.162.

2. Lamont RJ, Egland PG. Dental caries. In: Molecular medical microbiology. 2nd ed. Academic Press; 2015. p. 945-955. Available from: https://www.elsevier.com/books/molecular-medicalmicrobiology/tang/978-0-12-397169-2.

3. Evans CA, Kleinman DV, The surgeon general's report on America's oral health: opportunities for the dental profession. J Am Dent Assoc 2000;131(12):1721-1728. DOI: 10.14219/jada.archive.2000.0118.

4. Savarino L, Breschi L, Tedaldi M, et al. Ability of restorative and flouride releasing materials to prevent marginal dentine demineralization. Biomaterials 2004;25(6):1011-1017. DOI: 10.1016/s0142-9612(03)00628-8.

5. Gjorgievska E, Nicholson WJ, Iljovska S, et al. The potential of fluoridereleasing dental restoratives to inhibit enamel demineralization: an SEM study. Prilozi 2009;30(1):191-204. PMID: 19736541.

6. Joshi C, Gohil U, Parekh V, et al. Comparative evaluation of the remineralizing potential of commercially available agents on artificially demineralized human enamel: an in vitro study. Contemp Clin Dent 2019;10(4):605-613. DOI: 10.4103/ccd.ccd_679_18.

7. Chandru TP, Yahiya MB, Peedikayil FC, et al. Comparative evaluation of three different toothpastes on remineralization potential of initial enamel lesions: a scanning electron microscopic study. Indian J Dent Res 2020;31(2):217-223. DOI: 10.4103/ijdr.IJDR_745_18.

8. Freedman R, Diefenderfer KE. Effects of daily fluoride exposures on fluoride release by glass ionomer-based restoratives. Oper Dent 2003;28(2):178-185. PMID: 12670074.

9. Yli-Urpo H, Vallittu PK, Narhi TO, et al. Release of silica, calcium, phosphorus and fluoride from glass ionomer cement containing bioactive glass. J Biomater Appl 2004;19(1):5-20. DOI: $10.1177 / 0085328204044538$.

10. Hayacibara MF, Ambrozano GM, Cury JA. Simultaneous release of fluoride and aluminum from dental materials in various immersion media. Oper Dent 2004;29(1):16-22. PMID: 14753327.

11. Ulukapi H, Benderli Y, Soyman M. Determination of fluoride release from light-cured glass-ionomers and a fluoridated composite resin 
from the viewpoint of curing time. J Oral Rehabil 1996;23(3):197-201. DOI: 10.1111/j.1365-2842.1996.tb01233.x.

12. Baliga MS, Bhat SS. Effect of fluorides from various restorative materials on remineralization of adjacent tooth: an in vitro study. J Indian Soc Pedod Prev Dent 2010;28(2):84-90. DOI: 10.4103/0970-4388.66742.

13. Dionysopoulos $\mathrm{P}$, Kotsanos N, Koliniotou-Koubia, et al. Secondary caries formation in vitro around fluoride releasing restorations. Oper Dent 1994;19(5):183-188. PMID: 8700758.

14. Temin SC, Csuros Z, Mellberg JR. Fluoride uptake from a composite restorative by enamel. Dent Mater 1989;5(1):64-65. DOI: 10.1016/01095641(89)90096-1.

15. Wiegand A, Buchalla W, Attin T. Review on fluoridereleasing restorative materials-fluoride release and uptake characteristics, antibacterial activity and influence on caries formation. Dent Mater 2007;23(3):343362. DOI: 10.1016/j.dental.2006.01.022.

16. Preston AJ, Agalamanyi EA, Higham SM, et al. The recharge of esthetic dental restorative materials with fluoride in vitro two years' results. Dent Mater 2003;19(1):32-37. DOI: 10.1016/s0109-5641(02)00011-8.

17. Takahashi K, Emilson CG, Birkhed D. Fluoride release in vitro from various glass ionomer cements and resin composites after exposure to NaF solutions. Dent Mater 1993;9 (6):350-354. DOI: 10.1016/01095641(93)90055-u.
18. Albers HF. Resin ionomers. In: Tooth coloured restoratives: principles and techniques. 9th ed. BC Decker Inc; 2002. p. 57-67. Available from: https://www.worldcat.org/title/tooth-colored-restorativesprinciples-and-techniques/oclc/48417407.

19. DionysopoulosD,Koliniotou-KoumpiaE,Helvatzoglou-AntoniadesM Kotsanos N. In vitro inhibition of enamel demineralisation by fluoride-releasing restorative materials and dental adhesives. Oral Health Prev Dent 2016;14(4):371-380. DOI: 10.3290/j.ohpd. a35747.

20. Ikemura K, Tay FR, Kouro Y, et al. Optimizing filler content in an adhesive system containing pre-reacted glass-ionomer fillers. Dent Mater 2003;19(2):137-146. DOI: 10.1016/s0109-5641(02) 00022-2.

21. Tantbirojn D, Douglas WH, Versluis A. Inhibitive effect of a resin-modified glass ionomer cement on remote enamel artificial caries. Caries Res 1997;31(4):275-280. DOI: 10.1159/000262411.

22. Ferracane $\mathrm{JL}$, Mitchem JC, Adey JD. Fluoride penetration into the hybrid layer from a dentin adhesive. Am J Dent 1998;11(1):23-28. PMID: 9823082.

23. Yip HK, Smales RJ. Fluoride release from a polyacid-modified resin composite and 3 resin-modified glass-ionomer materials. Quintessence Int 2000;31(4):261-266. PMID: 11203934. 